M. Furushima and N. Nakayama

Nagoya Math. J.

Vol. 116 (1989), 111-122

\title{
THE FAMILY OF LINES ON THE FANO THREEFOLD $V_{5}$
}

\author{
MIKIO FURUSHIMA AND NOBORU NAKAYAMA
}

\section{Introduction}

A smooth projective algebraic 3 -fold $V$ over the field $C$ is called a Fano 3-fold if the anticanonical divisor $-K_{V}$ is ample. The integer $g=g(V)=\frac{1}{2}\left(-K_{V}\right)^{3}$ is called the genus of the Fano 3-fold $V$. The maximal integer $r \geqq 1$ such that $\mathcal{O}\left(-K_{V}\right) \cong \mathscr{H}^{r}$ for some (ample) invertible sheaf $\mathscr{H} \in \operatorname{Pic} V$ is called the index of the Fano 3 -fold $V$. Let $V$ be a Fano 3 -fold of the index $r=2$ and the genus $g=21$ which has the second Betti number $b_{2}(V)=1$. Then $V$ can be embedded in $\boldsymbol{P}^{6}$ with degree 5 , by the linear system $|\mathscr{H}|$, where $\mathcal{O}\left(-K_{V}\right) \cong \mathscr{H}^{2}$ (see Iskovskih [5]). We denote this Fano 3 -fold $V$ by $V_{5}$.

$V_{5}$ can be also obtained as the section of the Grassmannian $G(2,5)$ $\longrightarrow \boldsymbol{P}^{s}$ of lines in $\boldsymbol{P}^{4}$ by 3 hyperplanes in general position.

There are some other constructions of the Fano 3-fold $V_{5}$ (cf. Fujita [1], Mukai-Umemura [9] and Furushima-Nakayama [3]). But so obtained $V_{5}$ 's are all projectively equivalent (cf. [5]).

The remarkable fact on $V_{5}$ is that $V_{5}$ is a complex analytic compactification of $C^{3}$ which has the second Betti number one (see Problem 28 in Hirzebruch [4]).

Now, in this paper, we will analyze in detail the universal family of lines on $V_{5}$ and determine the hyperplane sections which can be the boundary of $C^{3}$ in $V_{5}$.

In $\S 1$, we will summarize some basic results about $V_{5}$ obtained by Iskovskih [5], Fujita [1] and Peternell-Schneider [6]. In $\S 2$, we will construct a $\boldsymbol{P}^{1}$-bundle $\boldsymbol{P}(\mathscr{E})$ over $\boldsymbol{P}^{2}$, where $\mathscr{E}$ is a locally free sheaf of rank 2 on $\boldsymbol{P}^{2}$, and a finite morphism $\psi: \boldsymbol{P}(\mathscr{E}) \rightarrow V_{5} \hookrightarrow \boldsymbol{P}^{6}$ of $\boldsymbol{P}(\mathscr{E})$ onto $V_{5}$ applying the results by Mukai-Umemura [9]. Further, we will show that the $\boldsymbol{P}^{1}$-bundle $\boldsymbol{P}(\mathscr{E})$ in fact the universal family of lines on $V_{5}$. In $\S 3$, we will study the boundary of $C^{3}$ in $V_{5}$ and the set $\left\{H \in\left|\mathcal{O}_{V}(1)\right| ; V_{5} \backslash H \cong C^{3}\right\}$.

Received March 1, 1988. 
Acknowledgement. The authors would like to thank the MaxPlanck-Institut für Mathematik in Bonn, especially, Prof. Dr. Hirzebruch for the hospitality and encouragement.

\section{§1. Basic facts on $V_{5}$}

Let $V:=V_{5}$ be a Fano 3 -fold of degree 5 in $P^{6}$ (see Introduction) and $\ell \cong P^{1}$ is a line on $V$. Then the normal bundle $N_{\ell \mid r}$ of $\ell$ in $V$ can be written as follows:

(a) $N_{\ell \mid V} \cong \mathcal{O}_{\ell} \oplus \mathcal{O}_{\ell}$, or

(b) $N_{\ell \mid V} \cong \mathcal{O}_{\ell}(-1) \oplus \mathcal{O}_{\ell}(1)$

We will call a line $\ell$ of the type $(0,0)$ (resp. $(-1,1)$ ) if $N_{\ell \mid V}$ is of the type (a) (resp. type (b)) above.

Let $\sigma: V^{\prime} \rightarrow V$ be the blowing up of $V$ along the line $\ell$, and put $L^{\prime}:=\sigma^{-1}(\ell)$. Then $L^{\prime} \cong P^{1} \times P^{1}$ if $\ell$ is of type $(0,0)$, and $L^{\prime} \cong F_{2}$ if $\ell$ is of type $(-1,1)$. Let $f_{1}, f_{2}$ be respectively fibers of the first and second projection of $\boldsymbol{P}^{1} \times \boldsymbol{P}^{1}$ onto $\boldsymbol{P}^{1}$, and let $s$, $f$ be respectively the negative section and a fiber of $F_{2}$. Let $H$ be a hyperplane section of $V$. Since the linear system $\left|\sigma^{*} H-L^{\prime}\right|$ on $V^{\prime}$ has no fixed component and no base point and since $h^{0}\left(\mathcal{O}\left(\sigma^{*} H-L^{\prime}\right)\right)=5$ and $\left(\sigma^{*} H-L^{\prime}\right)^{3}=\left(\sigma^{*} H-L^{\prime}\right)^{2} \cdot L^{\prime}=2$, the linear system $\left|\sigma^{*} H-L^{\prime}\right|$ defines a birational morphism $\varphi:=\varphi_{\left|\sigma^{*} H-L^{\prime}\right|}$ : $V^{\prime} \rightarrow W \smile P^{4}$ of $V^{\prime}$ onto a quadric hypersurface $W$ in $\boldsymbol{P}^{4}$, in particular, $Q:=\varphi\left(L^{\prime}\right)$ is a hyperplane section of $W$. Let $E:=E_{\ell}$ be the ruled surface swept out by lines which intersect the line $\ell$ and $E^{\prime}$ the proper transform of $E$ in $V^{\prime}$.

LEMMA 1.1 (Iskovskih [5], Fujita [1]). $W$ is a smooth quadric hypersurface in $P^{4}$ and $Y:=\varphi(E)$ is a twisted cubic curve contained in $Q$. In particular, $\varphi: V^{\prime} \rightarrow W$ is the blowing up of $W$ along the curve $Y$. Further, we have the following.

(a) If $\ell$ is of type $(0,0)$, then $\left.\varphi\right|_{L^{\prime}}: L^{\prime} \cong \boldsymbol{Q} \cong \boldsymbol{P}^{1} \times \boldsymbol{P}^{1}$, and $\bar{Y} \sim f_{1}+$ $2 f_{2}$ in $L^{\prime}$.

(b) If $\ell$ is of type $(-1,1)$, then $\left.\varphi\right|_{L^{\prime}}: L^{\prime} \rightarrow Q \cong \boldsymbol{Q}_{0}^{2}$ (a quadric cone) is the contraction of the negative section $s$ of $L^{\prime} \cong F_{2}$, and $\bar{Y} \sim s+3 f$ in $L^{\prime}$. $\bar{Y}$.

In (a) and (b), we denote the proper transform of $Y \subset Q$ in $L^{\prime}$ by

Corollary 1.1. (a) If $\ell$ is of type $(0,0)$, then $E^{\prime} \cong F_{1}$.

(b) If $\ell$ is of type $(-1,1)$, then $E^{\prime} \cong F_{3}$. 
Proof. Let $N_{Y \mid W}$ be the normal bundle of $Y$ in $W$. Then $N_{Y \mid W} \cong$ $\mathcal{O}_{Y}(3) \oplus \mathcal{O}_{Y}(4)$ if $\ell$ is of the type $(0,0)$, and $N_{Y \mid W} \cong \mathcal{O}_{Y}(2) \oplus \mathcal{O}_{Y}(5)$ if $Y$ is of type $(-1,1)$.

Q.E.D.

CoRollary 1.2. (a) If $l$ is of type $(0,0)$, then there are two points $q_{1} \neq q_{2}$ of $\ell$ such that (i) there are two lines in $V$ through the point $q_{i}$ $(i=1,2)$, and (ii) there are three lines in $V$ through every point $q$ of $\ell \backslash\left\{q_{1}, q_{2}\right\}$.

(b) If $\ell$ is of type $(-1,1)$, there is exactly one point $q_{0}$ of $\ell$ such that (i) $\ell$ is the unique line in $V$ through the point $q_{0}$, and (ii) there are two lines in $V$ through every point $q$ of $\ell \backslash\left\{q_{0}\right\}$.

Proof. (a) Let $p_{2}: Q \cong \boldsymbol{P}^{1} \times \boldsymbol{P}^{1} \rightarrow \boldsymbol{P}^{1}$ be the projection onto the second component. Since $\bar{Y} \sim f_{1}+2 f_{2},\left.p_{2}\right|_{Y}: Y \rightarrow P^{1}$ is a double cover over $\boldsymbol{P}^{1}$. Thus there are two branched point $b_{1} \neq b_{2}$ in $\boldsymbol{P}^{1}$. We put $q_{i}:=$ $\sigma \circ\left(\left.\varphi\right|_{L^{\prime}}\right)^{-1}\left(\left(\left.p_{2}\right|_{Y}\right)^{-1}\left(b_{i}\right)\right)(i=1,2)$. Then $\ell=\sigma(\bar{Y})$ and $\ell_{i}:=\sigma\left(\varphi^{-1}\left(p_{2}^{-1}\left(b_{i}\right)\right)(i=\right.$ $1,2)$ are two lines through the point $q_{i}$ for each $i$. For $b \in \boldsymbol{P}^{1} \backslash\left\{b_{1}, b_{2}\right\}$, $\ell=\sigma(\bar{Y})$ and $\sigma\left(\varphi^{-1}\left(p_{2}^{-1}(b)\right)\right)$ are three lines through the point $q \in \ell \backslash\left\{q_{1}, q_{2}\right\}$, since $p_{2}^{-1}(b)$ consists of two different points. This proves (a).

(b) We put $q_{0}:=\sigma(\bar{Y} \cap s) \in \ell$. Then $\ell=\sigma(\bar{Y})=\sigma(s)$ is the unique line through the point $q_{0} \in \ell$. For $y \in Y \backslash \varphi(s), \ell=\sigma(\bar{Y})$ and $\sigma\left(\varphi^{-1}(y)\right)$ are two lines through a point of $\ell \backslash\left\{q_{0}\right\}$. This proves (b).

Q.E.D.

Corollary 1.3 (Peternell-Schneider [6]). Let $E$ be a non-normal hyperplane section of $V_{5}$. Then the singular locus of $E$ is a line $\ell$ on $V$, in particular, $E$ is a ruled surface swept out by lines which intersect the line $\ell$. Further $V-E \cong C^{3}$ if and only if the line $\ell$ is of type $(-1,1)$.

Proof. By Lemma (3.35) in Mori [8], the non-normal locus of $E$ is a line $\ell$ on $V$. Since $h^{0}\left(\mathcal{O}_{V}(1) \oplus \mathscr{I}_{\ell}^{2}\right)=1$ and Pic $V \cong Z$, the linear system $\left|\mathcal{O}_{V}(1) \oplus \mathscr{I}_{\ell}^{2}\right|$ consists of $E$, where $\mathscr{I}_{\ell}$ is the ideal sheaf of $\ell$. By Lemma $1, \ell$ must be the singular locus of $E$. Assume $\ell$ is of type $(0,0)$. Then, by Lemma $1, V-E \cong\left\{(x, y, z, u) \in C^{4} ; x^{2}+y^{2}+z^{2}+u^{2}=1\right\} \not C^{3}$.

Q.E.D.

\section{§2. Construction of the universal family}

1. Let $(x: y),(u: v)$ be respectively homogeneous coordinates of the first factor and the second factor of $S:=\boldsymbol{P}^{1} \times \boldsymbol{P}^{1}$. Let us consider the diagonal $S L(2 ; C)$-action on $S$, namely, for $\sigma=\left(\begin{array}{ll}a & b \\ c & d\end{array}\right) \in S L_{2}:=S L(2 ; C)$, 


$$
\left\{\begin{array} { l } 
{ x ^ { \sigma } = a x + b y } \\
{ y ^ { \sigma } = c x + d y , }
\end{array} \quad \left\{\begin{array}{l}
u^{\sigma}=a u+b v \\
v^{\sigma}=c u+d v .
\end{array}\right.\right.
$$

Let $\tau: S \rightarrow \boldsymbol{P}^{2}$ be the double covering of $\boldsymbol{P}^{2}$ given by

$$
\left\{\begin{array}{l}
\tau^{*} X_{0}=x \otimes u \\
\tau^{*} X_{1}=\frac{1}{2}(x \otimes v+y \otimes u) \\
\tau^{*} X_{2}=y \otimes v
\end{array}\right.
$$

where $\left(X_{0}: X_{1}: X_{2}\right)$ be a homogeneous coordinate on $\boldsymbol{p}^{2}$. We can also define $S L_{2}$-action on $P^{2}$ as follows:

$$
\left\{\begin{array}{l}
X_{0}^{\sigma}=a^{2} X_{0}+2 a b X_{1}+b^{2} X_{2} \\
X_{1}^{\sigma}=a c X_{0}+(a d+b c) X_{1}+b d X_{2} \\
X_{2}^{\sigma}=c^{2} X_{0}+2 c d X_{1}+d^{2} X_{2}
\end{array}\right.
$$

for $\sigma=\left(\begin{array}{ll}a & b \\ c & d\end{array}\right) \in S L_{2}$.

Then, the morphism $\tau$ is $S L_{2}$-linear, that is, $\tau\left(p^{\sigma}\right)=\tau(p)^{\sigma}$ for $p \in S$ and $\sigma \in S L_{2}$. Further, $\tau$ is branched along the smooth conic $C:=\left\{X_{1}^{2}=X_{0} X_{2}\right\}=$ $\tau(\Delta)$, where $\Delta:=\Delta_{P^{1}}$ is the diagonal in $\boldsymbol{P}^{1} \times \boldsymbol{P}^{1}=S$. Let $f_{i}$ be a fiber of the projection $P_{i}: S \rightarrow \boldsymbol{P}^{1}$ onto $i$-th factor $(i=1,2)$. Let $\pi: M:=\boldsymbol{P}(\mathscr{E})$ $\rightarrow \boldsymbol{P}^{2}$ be the $\boldsymbol{P}^{1}$-bundle over $\boldsymbol{P}^{2}$ associated with the vector bundle $\mathscr{E}:=$ $\tau_{*} \mathcal{O}_{S}\left(4 f_{1}\right)$ of rank 2 on $\boldsymbol{P}^{2}$.

LEMMA 2.1. (1) $\operatorname{det}\left(\tau_{*} \mathcal{O}_{S}\left(k f_{1}\right)\right) \cong \mathcal{O}_{P^{2}}(k-1)$ and $c_{2}\left(\tau_{*} \mathcal{O}_{S}\left(k f_{1}\right)\right)=\frac{1}{2} k(k-1)$ for all $k \geqq 0$.

(2) $\mathscr{E} \otimes \mathcal{O}_{C} \cong \mathcal{O}_{\boldsymbol{P}_{1}}(3) \oplus \mathcal{O}_{\boldsymbol{P}_{1}}(3)$, where $C=\tau(\Delta)$.

(3) The natural morphism $S \rightarrow M$ corresponding to the homomorphism $\tau^{*} \mathscr{E} \rightarrow \mathcal{O}_{S}\left(4 f_{1}\right)$ is a closed embedding, hence, $S$ can be considered as a divisor on $M$.

(4) $\mathcal{O}_{M}(S) \cong \mathcal{O}_{\delta}(2) \otimes \pi^{*} \mathcal{O}_{P^{2}}(-2)$, where $\mathcal{O}_{\delta}(1)$ is the tautological line bundle on $M$ with respect to $\mathscr{E}$.

(5) $\mathcal{O}_{\mathscr{\delta}}(1)$ is nef, i.e., $\mathscr{E}$ is a semi-positive vector bundle

(6) We put $\mathcal{O}_{M}(1):=\mathcal{O}_{\ell}(1) \otimes \pi^{*} \mathcal{O}_{P^{2}}(1)$. Then

$$
\begin{aligned}
H^{0}\left(M, \mathcal{O}_{M}(1)\right) & \cong H^{0}\left(S, \mathcal{O}_{S}\left(5 f_{1}+f_{2}\right)\right) \\
& \cong H^{0}\left(\boldsymbol{P}^{1}, \mathcal{O}_{\boldsymbol{P}}(5)\right) \otimes_{C} H^{0}\left(\boldsymbol{P}^{1}, \mathcal{O}_{\boldsymbol{P}_{1}}(1)\right) .
\end{aligned}
$$

Proof. (1) Let us consider the exact sequence:

$$
0 \longrightarrow \tau_{*} \mathcal{O}_{S}\left(k f_{1}\right) \longrightarrow \tau_{*} \mathcal{O}_{S}\left((k+1) f_{1}\right) \longrightarrow \tau_{*} \mathcal{O} f_{1} \longrightarrow 0 .
$$


Now $\ell_{1}=\tau\left(f_{1}\right)$ is a line on $P^{2}$ and $\mathcal{O}_{\ell_{1}} \cong \tau_{*} \mathcal{O}_{1}$. Thus, $\operatorname{det}\left(\tau_{*} \mathcal{O}_{S}\left((k+1) f_{1}\right)\right)$ $\cong \operatorname{det}\left(\tau_{*} \mathcal{O}_{S}\left(k f_{1}\right)\right) \otimes \mathcal{O}(1) \quad$ and $\quad c_{2}\left(\tau_{*} \mathcal{O}_{S}\left((k+1) f_{1}\right)\right)=\left(\operatorname{det}\left(\tau_{*} \mathcal{O}_{S}\left(k f_{1}\right)\right) \cdot \mathcal{O}(1)\right)+$ $c_{2}\left(\tau_{*} \mathcal{O}_{\mathcal{S}}\left(\left(k f_{1}\right)\right)\right.$. Since $\tau_{*} \mathcal{O}_{\mathcal{S}} \cong \mathcal{O} \otimes \mathcal{O}(-1)$, we are done.

(2) Let us consider the following diagram:

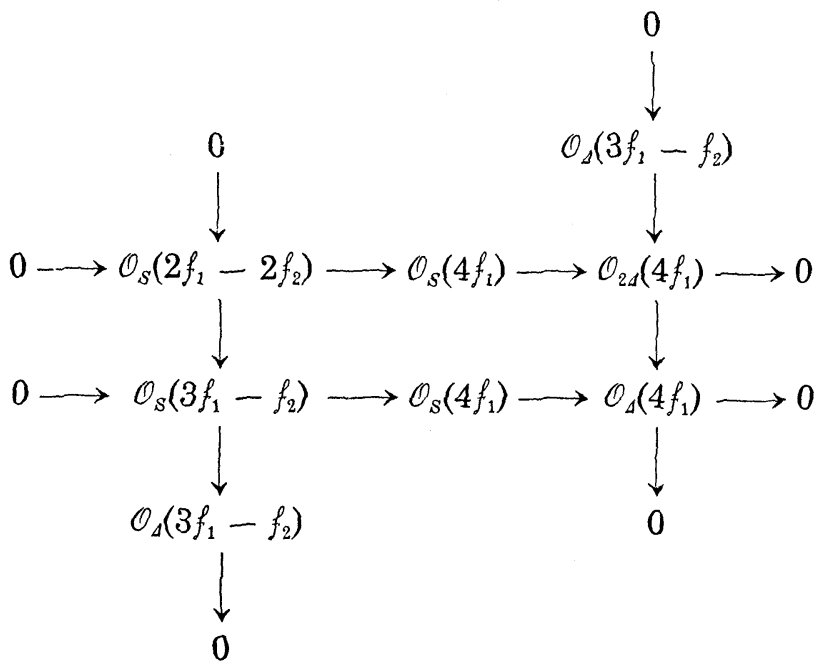

Since $\tau^{*} C=2 \Delta$, we have $\tau_{*} \mathcal{O}_{2 \Delta}\left(4 f_{1}\right) \cong \mathscr{E} \otimes \mathcal{O}_{C}$ and the exact sequence:

$$
\begin{gathered}
0 \longrightarrow \tau_{*} \mathcal{O}_{\Delta}\left(3 f_{1}-f_{2}\right) \longrightarrow \mathscr{E} \otimes \mathcal{O}_{C} \longrightarrow \tau_{*} \mathcal{O}_{\Delta}\left(4 f_{1}\right) \longrightarrow 0 \\
\mathcal{O}_{P_{1}}(2) \\
\mathcal{O}_{P 1}(4) .
\end{gathered}
$$

To show that $\mathscr{E} \otimes \mathcal{O}_{C} \cong \mathcal{O}_{P^{1}}(3) \oplus \mathcal{O}_{P^{1}}(3)$, it is enough to prove that

$$
H^{0}\left(C,\left(\mathscr{E} \otimes \mathcal{O}_{C}\right) \otimes \mathcal{O}_{\boldsymbol{P} 1}(-4)\right) \cong H^{0}\left(\mathcal{O}_{24}\left(2 f_{1}-2 f_{2}\right)\right)=0 \text {. }
$$

By the above diagram, we have the exact sequences:

$$
\begin{aligned}
& 0 \longrightarrow P_{2 *} \mathcal{O}_{\mathcal{S}}\left(-4 f_{2}\right) \stackrel{\varphi}{\longrightarrow} P_{2 *} \mathcal{O}_{\mathcal{S}}\left(2 f_{1}-2 f_{2}\right) \longrightarrow P_{2 *} \mathcal{O}_{24}\left(2 f_{1}-2 f_{2}\right) \longrightarrow 0, \\
& \text { III } 211 \\
& \mathcal{O}_{P_{1}}(-4) \quad \mathcal{O}_{P 1}(-2)^{\oplus 3}
\end{aligned}
$$

and

$$
\begin{aligned}
& 0 \longrightarrow P_{2^{*}} \mathcal{O}_{\Delta}\left(f_{1}-3 f_{2}\right) \longrightarrow P_{2 *} \mathcal{O}_{2 \triangleleft}\left(2 f_{1}-2 f_{2}\right) \longrightarrow P_{2 *} \mathcal{O}_{\Delta}\left(2 f_{1}-2 f_{2}\right) \longrightarrow 0 . \\
& \text { 2II } \\
& \mathcal{O}_{p 1}(-2)
\end{aligned}
$$

Hence $P_{2 *} \mathcal{O}_{2\lrcorner}\left(2 f_{1}-2 f_{2}\right)$ is locally free and the dual homomorphism $\varphi^{*}$ : $\mathcal{O}_{P_{1}}(2)^{\oplus 3} \rightarrow \mathcal{O}_{P_{1}}(4)$ is surjective. Therefore $\varphi^{*}$ is obtained from the natural 
surjection $H^{0}\left(\boldsymbol{P}^{1}, \mathcal{O}(2)\right) \otimes \mathcal{O}_{P_{1}} \longrightarrow \mathcal{O}_{P_{1}}(2)$ by tensoring $\mathcal{O}_{P_{1}}(2)$. Thus we have $P_{2 *} \mathcal{O}_{2 \Delta}\left(2 f_{1}-2 f_{2}\right) \cong \mathcal{O}_{P 1}(-1) \oplus \mathcal{O}_{P_{1}}(-1)$. Therefore we have $H^{0}\left(\mathcal{O}_{2 \Delta}\left(2 f_{1}-2 f_{2}\right)\right)$ $=0$.

(3) It is enough to show that the natural homomorphism $\operatorname{Sym}^{k} \mathscr{E} \rightarrow$ $\tau_{*} \mathcal{O}_{S}\left(4 k f_{1}\right)$ is surjective for $k \gg 0$. Since $\tau$ is finite morphism, $\tau_{*} \mathcal{O}_{S}\left(4 k f_{1}\right) \otimes$ $\tau_{*} \mathcal{O}_{S}\left(4 f_{1}\right) \rightarrow \tau_{*} \mathcal{O}_{S}\left(4(k+1) f_{1}\right)$ is always surjective. Thus we are done.

(4) Since $\tau: S \rightarrow \boldsymbol{P}^{2}$ is a double covering, there is a line bundle $\mathscr{L}$ on $\boldsymbol{P}^{2}$ such that $\mathcal{O}_{\delta}(2) \otimes \mathcal{O}_{M}(-S) \cong \pi^{*} \mathscr{L}$. By the exact sequence:

$$
0 \longrightarrow \pi^{*} \mathscr{L} \longrightarrow \mathcal{O}_{\delta}(2) \longrightarrow \mathcal{O}_{\delta}(2) \otimes \mathcal{O}_{S} \cong \mathcal{O}_{S}\left(8 f_{1}\right) \longrightarrow 0,
$$

we have $\operatorname{det}\left(\operatorname{Sym}^{2} \mathscr{E}\right) \cong \mathscr{L} \otimes \operatorname{det}\left(\tau_{*} \mathcal{O}_{S}\left(8 f_{1}\right)\right)$. Therefore, by $(1), \mathscr{L} \cong \mathcal{O}_{p^{2}}(2)$, hence, $\mathcal{O}_{M}(S) \cong \mathcal{O}_{\delta}(2) \otimes \pi^{*} \mathcal{O}_{P^{2}}(-2)$.

(5) We put $D:=\pi^{-1}(C)$. Then, by (2), $D \cong \boldsymbol{P}^{1} \times \boldsymbol{P}^{1}$ and $\mathcal{O}_{8}(1) \otimes \mathcal{O}_{D}$ $\cong \mathcal{O}_{D}\left(s_{1}+3 s_{2}\right)$, where $s_{2}$ is a fiber of $D \rightarrow C$ and $s_{1}$ is a fiber of another projection $D \rightarrow P^{1}$. By (4), we have $\mathcal{O}_{\delta}(2) \cong \mathcal{O}_{M}(S+D)$. Assume that there is an irreducible curve $\gamma$ on $M$ such that $\left(\mathcal{O}_{s}(1) \cdot \gamma\right)<0$. Then, $\gamma \subseteq D$ or $\gamma \subseteq S$. Since $\mathcal{O}_{\delta}(1) \otimes \mathcal{O}_{S} \cong \mathcal{O}_{S}\left(4 f_{1}\right)$ and $\mathcal{O}_{\delta}(1) \otimes \mathcal{O}_{D} \cong \mathcal{O}_{D}\left(s_{1}+3 s_{2}\right)$, this is a contradiction.

(6) By the exact sequence

$$
\begin{aligned}
& 0 \longrightarrow \mathcal{O}_{M}(1) \otimes \mathcal{O}_{M}(-S) \longrightarrow \mathcal{O}_{M}(1) \longrightarrow \mathcal{O}_{M}(1) \otimes \mathcal{O}_{S} \longrightarrow 0, \\
& \text { 2II } \\
& \mathcal{O}_{\varepsilon}(-1) \otimes \pi^{*} \mathcal{O}_{\boldsymbol{P}^{2}}(3) \quad \mathcal{O}_{S}\left(5 f_{1}+f_{2}\right)
\end{aligned}
$$

we have $\pi_{*} \mathcal{O}_{M}(1) \cong \tau_{*} \mathcal{O}_{S}\left(5 f_{1}+f_{2}\right)$. Therefore $H^{0}\left(M, \mathcal{O}_{M}(1)\right) \cong H^{0}\left(S, \mathcal{O}_{S}\left(5 f_{1}+f_{2}\right)\right)$.

Q.E.D.

Remark 2.1. There is a $S L_{2}$-action on $\left(M, \mathcal{O}_{M}(1)\right)$ compatible to $\tau: S \rightarrow$ $\boldsymbol{P}^{2}$. The last isomorphism in (6) is an isomorphism as a $S L_{2}$-module.

2. Let us consider the subvector space $L \subseteq H^{0}\left(S, \mathcal{O}_{S}\left(5 f_{1}+f_{2}\right)\right)$ generated by the following 7 elements (cf. Lemma (1.6) in [9]):

$$
\left\{\begin{array}{l}
e_{0}:=x^{5} \otimes u \\
e_{1}:=x^{4} y \otimes u+\frac{1}{5} x^{5} \otimes v \\
e_{2}:=x^{3} y^{2} \otimes u+\frac{1}{2} x^{4} y \otimes v \\
e_{3}:=x^{2} y^{3} \otimes u+x^{3} y^{2} \otimes v \\
e_{4}:=\frac{1}{2} x y^{4} \otimes u+x^{2} y^{3} \otimes v \\
e_{5}:=\frac{1}{5} y^{5} \otimes u+x y^{4} \otimes v \\
e_{6}:=y^{5} \otimes v
\end{array}\right.
$$


Then $L$ is an $S L_{2}$-invariant subspace. By the isomorphism $H^{\circ}\left(M, \mathcal{O}_{M}(1)\right)$ $\cong H^{0}\left(S, \mathcal{O}_{S}\left(5 f_{1}+f_{2}\right)\right), L$ can be considered as a subspace of $H^{0}\left(M, \mathcal{O}_{M}(1)\right)$.

Lemma 2.2. (1) The homomorphism $L \otimes \mathcal{O}_{M} \rightarrow \mathcal{O}_{M}(1)$ is surjective. Especially, we have a morphism $\psi: M \rightarrow \boldsymbol{P}(L) \cong \boldsymbol{P}^{6}$, which is $S L_{2}$-linear.

(2) The image $V:=\psi(M)$ is isomorphic to the Fano 3-fold $V_{5}$ of de. gree 5 in $\boldsymbol{P}^{6}$.

Proof. (1) We have only to show that $g: L \otimes \mathcal{O}_{P^{2}} \rightarrow \mathscr{E} \otimes \mathcal{O}_{P^{2}}(1)$ is surjective. Since $S L_{2}$ acts on $g$, the support of Coker $(g)$ is $S L_{2}$-invariant. Now $S L_{2}$ acts on $\boldsymbol{P}^{2}$ with two orbits $\boldsymbol{P}^{2} \backslash C$ and $C$. First, take a point $p \in \boldsymbol{P}^{2} \backslash C$. Then $g \otimes C(p): L \rightarrow\left(\mathscr{E} \otimes \mathcal{O}_{\boldsymbol{P}^{2}}(1)\right) \otimes C(p)$ is described as follows:

Let $\alpha: L \otimes \mathcal{O}_{S} \rightarrow \mathcal{O}_{S}\left(5 f_{1}+f_{2}\right)$ be the natural homomorphism and let $\alpha(q): L \rightarrow \mathcal{O}_{S}\left(5 f_{1}+f_{2}\right) \otimes C(q) \cong C$ be the evaluation map for $q \in S$. Then $g \otimes C(p): L \rightarrow C^{\oplus 2}$ is nothing but $\alpha\left(q_{1}\right) \oplus \alpha\left(q_{2}\right): L \rightarrow C^{\oplus 2}$, where $\left\{q_{1}, q_{2}\right\}:=$ $\tau^{-1}(p)$. For example, take a point $p=(0: 1: 0) \in \boldsymbol{P}^{2}$. Then $q_{1}=((1: 0)$, $(0: 1))$ and $q_{2}=((0: 1),(1: 0))$ in $S=\boldsymbol{P}^{1} \times \boldsymbol{P}^{1}$. Then the calculation is as follows:

$$
\begin{cases}\alpha_{1}\left(e_{0}\right)=\alpha_{1}\left(e_{2}\right)=\cdots=\alpha_{1}\left(e_{6}\right)=0, & \alpha_{1}\left(e_{1}\right)=\frac{1}{5} \\ \alpha_{2}\left(e_{0}\right)=\cdots=\alpha_{2}\left(e_{4}\right)=\alpha_{2}\left(e_{5}\right)=0, & \alpha_{2}\left(e_{5}\right)=\frac{1}{5},\end{cases}
$$

where $\alpha_{1}:=\alpha_{1}\left(q_{1}\right), \alpha_{2}:=\alpha_{2}\left(q_{2}\right)$.

Therefore $g \otimes C(p)$ is surjective for any $P \in \boldsymbol{P}^{2} \backslash C$.

Next take $p:=(1: 0: 0) \in C, q=((1: 0),(1: 0)) \in S$. Let $z_{1}=y / x, z_{2}=$ $v / u$ be the local coordinate around $q$. Then $\mathfrak{m}_{p} \mathcal{O}_{S}=\left(z_{1}+z_{2}, z_{1} \cdot z_{2}\right) \subseteq \mathfrak{m}_{q}$. The evaluation map $q \otimes C(p): L \rightarrow C^{\oplus 2}$ is now the composition

$$
\beta: L \longrightarrow L \otimes \mathcal{O}_{S} \longrightarrow \mathcal{O}_{S} / \mathfrak{m}_{p} \mathcal{O}_{S} \cong C 1 \oplus C \bar{z}_{1} .
$$

Since we have isomorphisms

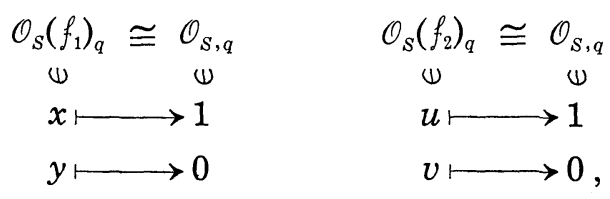

$\beta: g \otimes C(p)$ is calculated by evaluating $x=u=1$ and $y=\bar{z}_{1}=-v=-\bar{z}_{2}$. Therefore $\beta\left(e_{0}\right)=1, \quad \beta\left(e_{1}\right)=\frac{4}{5} \bar{z}_{1}, \quad \beta\left(e_{2}\right)=0, \quad \beta\left(e_{3}\right)=0, \quad \beta\left(e_{4}\right)=0, \quad \beta\left(e_{5}\right)=0$, $\beta\left(e_{6}\right)=0$. Thus $g \otimes C(p)$ is surjective for any $p \in C$.

(2) Let $h_{0}, h_{1}, \cdots, h_{6} \in L^{\vee}$ be the dual basis of $\left\{e_{0}, e_{1}, \cdots, e_{6}\right\}$. Since $\boldsymbol{P}(L) \cong L^{\vee} \backslash\{0\} / \boldsymbol{C}^{*}$, we denote the point of $\boldsymbol{P}(L)$ corresponding to $\sum_{j=0}^{6} \lambda_{j} h_{j}$ 
$\in L^{\bigvee} \backslash\{0\}$ by $\left[\sum_{j=0}^{6} \lambda_{j} h_{j}\right]$. If $\psi(M)$ contains the point $\left[h_{1}-h_{5}\right] \in \boldsymbol{P}(L)$, then $\psi(M)$ contains the $S L_{2}$-orbit $S L_{2}\left[h_{1}-h_{5}\right]$ and its closure $\overline{S L_{2}\left[h_{1}-h_{5}\right]}$. On the other hand, we know that the closure $\overline{S L_{2}\left[h_{1}-h_{5}\right]}$ is isomorphic to $V_{5}$ by $[\S 3,7]$. Here $h_{1}-h_{5}$ corresponds to $f_{6}(x, y)=x y\left(x^{4}-y^{4}\right)$ in their notation. Therefore we have only to show that $\psi(M)$ contains $\left[h_{1}-h_{5}\right]$ $\in \boldsymbol{P}(L)$. Let $P:=(0: 1: 0) \in \boldsymbol{P}^{2}$. Then by (1), the evaluation map $\boldsymbol{g} \otimes$ $C(p): L \rightarrow C \oplus C$ with $(g \otimes C(p))\left(e_{1}\right)=\left(\frac{1}{5}, 0\right), \quad(g \otimes C(p))\left(e_{5}\right)=\left(0, \frac{1}{5}\right)$, and $(g \otimes C(p))\left(e_{j}\right)=(0,0)(j \neq 1,5)$. Therefore the point $q \in \pi^{-1}(p) \cong P^{1}$ corresponding to the linear function $C \oplus C \ni(a, b) \mapsto a-b \in C$ is mapped to $\left[h_{1}-h_{5}\right]$ by $\psi$.

Q.E.D.

Remark 2.2. (1) By Lemma (1.5) in [8], $V:=\psi(M)$ has three $S L_{2^{-}}$ orbits $\psi(M) \backslash \psi(S), \psi(S) \backslash \psi\left(\Delta_{P_{1}}\right)$, and $\psi\left(\Delta_{P_{1}}\right)$, in particular, $\psi\left(\Delta_{P_{1} 1}\right)$ is a smooth rational curve of degree 6 in $V$.

(2) $\left.\psi\right|_{s}: S \rightarrow \psi(S)$ is the same morphism as in Lemma (1.6) in [8]. Especially, $\left.\psi\right|_{S}$ is one to one and $\operatorname{Sing} \psi(S)=\psi\left(\Delta_{P_{1}}\right)$, where Sing $\psi(S)$ is the singular locus of $\psi(S)$.

Let us denote $\psi(S)$ and $\psi\left(\Delta_{P 1}\right)$ by $B$ and $\Sigma$.

LEMma 2.3. (1) $\psi$ is a finite morphism of degree 3.

(2) $\psi$ is étale outsides $B$

(3) $\psi^{*} B=S+2 D$, hence $\psi$ is not Galois.

(4) We put $M_{t}:=\pi^{-1}(t)$ for $t \in \boldsymbol{P}^{2}$. Then $\ell_{t}:=\psi\left(M_{t}\right)$ is a line of $V \cong P^{6}$ and $\left.\psi\right|_{M_{t}}: M_{t} \rightarrow \ell_{t}$ is an isomorphism.

(5) For $t_{1} \neq t_{2} \in \boldsymbol{P}^{2}$, we have $\ell_{t_{1}} \neq \ell_{t_{2}}$.

(6) Let $\ell$ be a line in $V \cong \boldsymbol{P}^{6}$. Then there is a point $t \in \boldsymbol{P}^{2}$ such that $\ell=\ell_{t}$.

Proof. (1) By Lemma (2.1)-(5), $\mathcal{O}_{M}(1)$ is ample. Therefore $\psi$ is a finite morphism and $\psi^{*} \mathcal{O}_{V}(1) \cong \mathcal{O}_{M}(1)$. Thus $\operatorname{deg} \psi=\left(\mathcal{O}_{M}(1)\right)^{3} /\left(\mathcal{O}_{V}(1)\right)^{3}=15 / 5$ $=3$.

(2) Since $V \backslash B$ is an open orbit of $S L_{2}, \psi$ is étale over $V-B$.

(3) Since $\left(\mathcal{O}_{V}(1)^{2} \cdot B\right)=\left(\mathcal{O}_{M}(1)^{2} \cdot S\right)=\left(\mathcal{O}_{S}\left(5 f_{1}+f_{2}\right)\right)_{S}^{2}=10$, we have $\mathscr{O}_{V}(B)$ $\mathcal{O}_{V}(2)$. Therefore $\mathcal{O}_{M}\left(\psi^{*} B-S\right) \cong \pi^{*} \mathcal{O}_{P^{2}}(4)$. Since $\psi^{*} B-S$ is a $S L_{2^{-}}$ invariant effective divisor, its support must be $D$. Thus $\psi^{*} B=S+2 D$.

(4) It is clear since $\left(\psi^{*} \mathcal{O}_{V}(1) \cdot M_{t}\right)=\left(\mathcal{O}_{M}(1) \cdot M_{t}\right)=1$.

(5) Assume that $\ell_{t_{1}}=\ell_{t_{2}}$. Since $\left.\psi\right|_{s}: S \rightarrow B$ is one to one, we have $M_{t_{1}} \cap S=M_{t_{2}} \cap S$. Hence $t_{1}=t_{2}$.

(6) Let $\ell$ be a line of $V$. If $\ell \not \subset B$, then $\ell$ contains a point $p \in V \backslash B$. 
By Corollary (1.2) in $\S 1$, we have $\#\{$ lines through $p\} \leqq 3$. Thus by (4), (5) above, \{lines through $p\}=\left\{\ell_{t_{1}}, \ell_{t_{2}}, \ell_{t_{3}}\right\}$, where $\left\{t_{1}, t_{2}, t_{3}\right\}=\pi\left(\psi^{-1}(p)\right)$. Therefore $\ell=\ell_{t_{2}}$. If $\ell \leqq B$, then $\ell=\ell_{t}$ for some $t \in C$, because $\left.\psi\right|_{D}$ : $D \rightarrow B$ is one to one by (3) and $\mathcal{O}_{M}(1) \otimes \mathcal{O}_{D} \cong \mathcal{O}_{D}\left(s_{1}+5 s_{2}\right)$ by Lemma 2.1(2).

Theorem I. The $\boldsymbol{P}^{1}$-bundle $\pi: M \rightarrow \boldsymbol{P}^{2}$ is the universal family of lines on $V=V_{5}$.

Proof. Let $T$ be the space of lines on $V$, that is, $T$ is a subscheme of the Grassmannian $G(2,7)$ parametrizing lines of $V \cong \boldsymbol{P}^{6}$. Since $N_{\ell \mid V} \cong$ $\mathcal{O} \oplus \mathcal{O}$ or $\mathcal{O}(-1) \oplus \mathcal{O}(1)$ for any line $\ell$ on $V$, we have $H^{1}\left(\ell, N_{\ell \mid V}\right)=0$ and $H^{0}\left(\ell, N_{\ell \mid V}\right) \cong C^{2}$. Therefore $T$ is smooth surface. By the universal property of $T$, we have a morphism $\delta: P^{2} \rightarrow T$ corresponding to the family $(\pi, \psi): M \smile P^{2} \times V$. By Lemma (1.3)-(5), (6), $\delta$ is one to one surjective. Therefore $\delta$ must be isomorphic.

We put $U_{n}:=\{x \in V$; there is at most $n$ lines through $x\}$. Then,

Corollary 2.1. $U_{3}=V, U_{2}=B$ and $U_{1}=\Sigma$.

\section{§3. Compactifications of $C^{3}$}

Take any point $t \in C \longrightarrow P^{2}$ and put $\ell_{t}:=\psi\left(\pi^{-1}(t)\right)$. Then $\ell_{t}$ is a line of type $(-1,1)$. Let $\sigma: V^{\prime} \rightarrow V$ be blowing up of $V$ along the line $\ell_{t}$ and $\bar{E}_{t}$ be the proper transform in $V^{\prime}$ of the ruled surface $E_{t}$ swept out by lines which intersect the line $\ell_{t}$. Then, by Lemma 1.1-(b), we have the birational morphism $\varphi: V^{\prime} \rightarrow W_{t}$ of $V^{\prime}$ onto a smooth quadric hypersurface $W_{t} \cong \boldsymbol{Q}^{3}$ in $\boldsymbol{P}^{4}$, a quadric cone $\boldsymbol{Q}_{t}:=\varphi\left(\sigma^{-1}\left(\ell_{t}\right)\right) \cong \boldsymbol{Q}_{0}^{2}$, and a twisted cubic curve $Y_{t}:=\varphi\left(\bar{E}_{t}\right) \longrightarrow Q_{t}$. Let $g_{t}$ be the unique generating line of $Q_{t}$ such that $Y_{t} \cap g_{t}=\left\{v_{t}\right\}$, where $v_{t}$ is the vertex of $Q_{t}$. Take any point $v \in g_{t} \backslash\left\{v_{t}\right\} \cong C$. Let $Q_{v}$ be the quadric cone in $W_{t}$ with the vertex $v$, and put $H_{t}^{v}:=\sigma\left(\varphi^{-1}\left(Q_{v}\right)\right)$.

Then, by (4.3) in [2] and [6] (see also §1), we have the following

Lemma 3.1. (1) For any $t \in C,\left(V, E_{t}\right)$ is a compactification of $C^{3}$ with the non-normal boundary $E_{t}$. Conversely, let $(V, H)$ be a compactification of $C^{3}$ with a non-normal boundary $H$. Then there is a point $t \in C$ such that $H=E_{t}$.

(2) For any $t \in C$ and any $v \in g_{t} \backslash\left\{v_{t}\right\} \cong C$, $\left(V, H_{t}^{v}\right)$ is a compactification of $C^{3}$ with the normal boundary $H_{t}^{v}$. Conversely, let $(V, H)$ be a com- 
pactification of $C^{3}$ with a normal boundary $H$. Then there is a point $t \in C$ and a point $v \in g_{t} \backslash\left\{v_{t}\right\}$ such that $H=H_{t}^{v}$.

Remark 3.1. Let $Z_{t}$ be the line $\boldsymbol{P}^{2}$ which is tangent to $C$ at the point $t \in C$. Then $E_{t}=\psi\left(\pi^{-1}\left(Z_{t}\right)\right)$ and $\pi^{-1}\left(Z_{t}\right) \backslash\left(s_{t} \cup \pi^{-1}(t)\right) \cong E_{t} \backslash \ell_{t}$, where $s_{t}$ is the negarive section of $\pi^{-1}\left(Z_{t}\right) \cong F_{3}$.

We put

$\Lambda_{1}:=\left\{\lambda \in \check{\boldsymbol{P}}^{6} ; H_{\lambda}\right.$ is a non-normal hyperplane section of $V$ such that $\left.V \backslash H_{\lambda} \cong C^{3}\right\}$, and

$\Lambda_{2}:=\left\{\lambda \in \check{\boldsymbol{P}}^{6} ; H_{\lambda}\right.$ is a normal hyperplane section of $V$ such that $V \backslash H_{i}$ $\left.\cong C^{3}\right\}$

where $\check{\boldsymbol{P}}^{\bullet}:=\boldsymbol{P}(\check{L})$.

Then we have

Corollary 3.1. $\operatorname{dim}_{C} \Lambda_{1}=1$ and $\operatorname{dim}_{C} \Lambda_{2}=2$.

Corollary 3.2. For each $t \in C, \quad\left\{\lambda \in \Lambda_{1} ; \ell_{t} \leqq H_{\lambda}\right\}=\{$ one point $\}$ and $\left\{\lambda \in \Lambda_{2} ; \ell_{t} \subseteq H_{\lambda}\right\} \cong C$.

Now, take a point $t_{0}=(1: 0: 0) \in C$. Then $\ell_{t_{0}} \longrightarrow P^{6}$ is written as follows:

$$
\ell_{t_{0}}=\left\{h_{2}=h_{3}=h_{4}=h_{5}=h_{6}=0\right\}
$$

(see the proof of Lemma 2.2-(1)).

Since $V$ is $S L_{2}$-invariant, $\Lambda_{1}$ and $\Lambda_{2}$ are also $S L_{2}$-invariant

By Lemma (1.4) of [9], the 2-dimensional $S L_{2}$-orbits are $S L_{2} x^{3} y^{3}$, $S L_{2} x^{4} y^{2}=S L_{2} x^{2} y^{4}, S L_{2} x^{5} y=S L_{2} x y^{5}$, and further $S L_{2} y^{6}=S L_{2} x^{6}$ is the only one $S L_{2}$-orbit of dimension one on $\boldsymbol{P}^{6}$. Therefore we have $\Lambda_{1}=S L_{2} y^{6}$. By an easy calculation, we have

$$
\begin{aligned}
& \left\{\lambda \in S L_{2} x^{3} y^{3} ; \ell_{t_{0}} \subseteq H_{\lambda}\right\} \cong C \cup C, \\
& \left\{\lambda \in S L_{2} x^{2} y^{4} ; \ell_{t_{0}} \cong H_{\lambda}\right\} \cong C \cup C, \\
& \left\{\lambda \in S L_{2} x y^{5} ; \ell_{t_{0}} \cong H_{\lambda}\right\} \cong C .
\end{aligned}
$$

Thus, by Corollary 3.2, we must have $\Lambda_{2}=S L_{2} x y^{5}$. We put $\Lambda:=\Lambda_{1}$ $\cup \Lambda_{2}$. Then $\Lambda=\overline{S L_{2} x y^{5}}$. Therefore, by Lemma (1.6) of [9], $\Lambda$ is the image of $\boldsymbol{P}^{1} \times \boldsymbol{P}^{1}$ with diagonal $S L_{2}$-operations by a linear system $L$ of bidegree $(5,1)$ on $\boldsymbol{P}^{1} \times \boldsymbol{P}^{1}$.

Thus we have

THEOREM 3.1. $\Lambda_{1}=S L_{2} y^{6}, \Lambda_{2}=S L_{2} x y^{5}$ and $\Lambda=\overline{S^{\prime} L_{2} x y^{5}}$. In particular, $\Lambda_{1} \cong \boldsymbol{P}^{1}$ and $\Lambda_{2} \cong \boldsymbol{P}^{1} \times \boldsymbol{P}^{1} \backslash\{$ diagonal $\}$. 
We will show explicitly below that for any $\lambda \in \Lambda, V \backslash H_{2} \cong C^{3}$.

By p. 505 in [9], $V:=V_{5} \longrightarrow \boldsymbol{P}^{6}$ can be written as follows:

$$
\left\{\begin{array}{l}
h_{0} h_{4}-4 h_{1} h_{3}+3 h_{2}^{2}=0 \\
h_{0} h_{5}-3 h_{1} h_{4}+2 h_{2} h_{3}=0 \\
h_{0} h_{6}-9 h_{2} h_{4}+8 h_{3}^{2}=0 \\
h_{1} h_{6}-3 h_{2} h_{5}+2 h_{3} h_{4}=0 \\
h_{2} h_{6}-4 h_{3} h_{5}+3 h_{4}^{2}=0
\end{array}\right.
$$

where $\left(h_{0}: h_{1}: h_{2}: h_{3}: h_{4}: h_{5}: h_{6}\right)$ is the homogeneous coordinate of $\boldsymbol{P}^{6}$.

We have $(0: 0: 0: 0: 0: 0: 1) \in S L_{2} y^{6}$. In $V \cap\left\{h_{6} \neq 0\right\}$, we consider the following coordinate transformation

Then we have

$$
\left\{\begin{array}{l}
x_{0}=h_{0}-9 h_{2} h_{4}+8 h_{3}^{2} \\
x_{1}=h_{1}-3 h_{2} h_{5}+3 h_{3} h_{4} \\
x_{2}=h_{2}-4 h_{3} h_{5}+3 h_{4}^{2} \\
x_{3}=h_{3} \\
x_{4}=h_{4} \\
x_{5}=h_{5} \\
h_{6}=1 .
\end{array}\right.
$$

$$
V \cap\left\{h_{6} \neq 0\right\} \cong\left\{x_{0}=x_{1}=x_{2}=0\right\} \cong C^{3},
$$

and the line $\left\{h_{2}=h_{3}=h_{4}=h_{5}=h_{6}=0\right\}$ is the singular locus of the boundary $V \cap\left\{h_{6}=0\right\}$.

We have $(0: 0: 0: 0: 0: 1: 0) \in S L_{2} x y^{5}$. In $V \cap\left\{h_{5} \neq 0\right\}$, we consider the coordinate transformation

$$
\left\{\begin{array}{l}
x_{0}=h_{0}-3 h_{1} h_{4}+2 h_{2} h_{3} \\
x_{1}=h_{1} \\
x_{2}=3 h_{2}-h_{1} h_{6}-2 h_{3} h_{4} \\
x_{3}=4 h_{3}-h_{2} h_{6}-3 h_{4}^{2} \\
x_{4}=h_{4} \\
x_{6}=h_{6} \\
h_{5}=1 .
\end{array}\right.
$$

Then we have

$$
V \cap\left\{h_{5} \neq 0\right\} \cong\left\{x_{0}=x_{2}=x_{3}=0\right\} \cong C^{3},
$$

and the boundary $V \cap\left\{h_{5}=0\right\}$ has a singularity of $A_{4}$-type at the point 
(1:0:0:0:0:0:0).

Therefore, for any $\lambda \in S L_{2} y^{6}$ (resp. $S L_{2} x y^{5}$ ), $H_{\lambda}$ is non-normal (resp. normal with a rational double point of $A_{4}$-type), and further $V \backslash H_{2} \cong C^{3}$.

Since $\Lambda_{1}$ and $\Lambda_{2}$ are $S L_{2}$-orbits, we have the following

Corollary 3.3 (cf. [6]). Let $(V, H)$ and $\left(V, H^{\prime}\right)$ be two compactifications of $C^{3}$ with normal (resp. non-normal) boundaries $H$ and $H^{\prime}$. Then there is an automorphism $\alpha$ of $V$ such that $H^{\prime}=\alpha(H)$.

\section{REFERENCES}

[1] T. Fujita, On the structure of polarized manifolds with total deficiency one, II, J. Math. Soc. Japan, 33 (1981), 415-434.

[2] M. Furushima, Singular del Pezzo surfaces and analytic compactifications of 3dimensional complex affine space $C^{3}$, Nagoya Math. J., 104 (1986), 1-28.

[3] N. Furushima - N. Nakayama, A new construction of a compactification of $C^{3}$, Tôhoku Math. J., 41 (1989), 543-560.

[4] F. Hirzebruch, Some problems on differentiable and complex manifolds, Ann. Math., 60 (1954), 213-236.

[ 5 ] V. A. Iskovskih, Fano 3-fold I, Math. U.S.S.R. Izvestija, 11 (1977), 485-527.

[6] Th. Peternell - M. Schneider, Compactifications of $C^{3}(\mathrm{I})$, Math. Ann., 280 (1988), $129-146$.

[7] M. Miyanishi, Algebraic methods in the theory of algebraic threefolds, Advanced study in Pure Math. 1, 1983 Algebraic varieties and Analytic varieties, 66-99.

[ 8 ] S. Mori, Threefolds whose canonical bundle are not numerical effective, Ann. Math., 116 (1982), 133-176.

[9] S. Mukai-H. Umemura, Minimal rational threefolds, Lecture Notes in Math., 1016, Springer-Verlag (1983), 490-518.

Max-Planck-Institut

für Mathematik

Gottfried-Claren-Str. 26

5300 Bonn 3

West Germany

Current address

M. Furushima

Department of Mathematics

Faculty of Education

Ryukyu University

Okinawa, 903-01

Japan

N. Nakayama

Department of Mathematics

Faculty of Science

University of Tokyo

Hongo, Tokyo, 113

Japan 